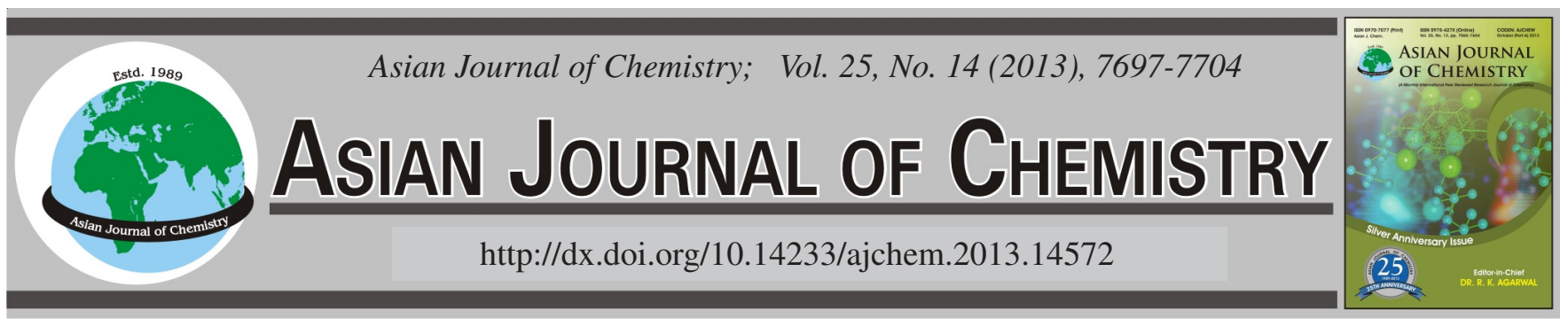

\title{
Identification of Related Substances in Citicoline Sodium Injection by HPLC-DAD and HPLC-MS/MS
}

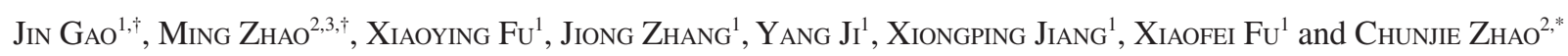

${ }^{1}$ Institute for Drug and Instrument Control of Health Department GLD of PLA, No. 17 Fengtai West Road, Beijing 100071, P.R. China ${ }^{2}$ School of Pharmacy, Shenyang Pharmaceutical University, No. 103 Wenhua Road, Shenyang 110016, P.R. China

${ }^{3}$ Clinical Pharmaceutics Room, The People's Hospital of Wenshan Prefecture, No. 228 Kaihua Middle Road, Wenshan 663000, P.R. China

*Corresponding author: Fax: +86 24 23986250; Tel: +86 24 23986299; E-mail: chenlei00000@126.com

$\uparrow$ These authors have equal contribution to this work. Both persons are the first authors.

\begin{abstract}
In the present study a method based on liquid chromatography with diode array detection (HPLC-DAD) coupled to an electrospray ionization (ESI) interface for identify the chemical structures of related substances in citicoline sodium injection was established. There are seven impurities in a typical chromatogram of citicoline sodium injection (5'-CMP has known). The UV spectrum and molecular weight of related substances were obtained and the chemical structures were presumed according to production process. Compared with reference substances, two impurities were confirmed as cytosine and cytidine. As for the largest related substance (except 5'-CMP), it was collected by liquid chromatography and treated by solid-phase extraction for LC-MS system. Compared with self made reference substance, it was confirmed as uridine diphosphate choline. The three main related substances were identified in citicoline sodium injection for the first time.
\end{abstract}

Key Words: HPLC-DAD, HPLC-MS/MS, Citicoline sodium injection, Related substances, Identification.

\section{INTRODUCTION}

Citicoline administered exogenously provides both choline and cytidine, which were served as substrates for the synthesis of phosphatidylcholine, a primary component of neuronal membrane. It is believed to have promoted membrane synthesis and repair, which are essential for recovery from neuronal injury ${ }^{1-4}$. A neuroprotective effect of citicoline has been shown in clinical studies of citicoline treated older subjects in whom cognitive and behavioural parameters improved $^{5,6}$. In patients with stroke, citicoline improves neurological outcomes and reduces the volume of the ischemic injury ${ }^{7-15}$. Citicoline sodium injection was recorded in ChP2010 $0^{16}$ and was used widely in clinical practice. The related substances of citicoline sodium injection were analyzed by HPLC-UV method in ChP2010 and seven impurities (named as impurity $\mathrm{A} \sim \mathrm{G}$ ) were found in a typical chromatogram in which only one impurity was confirmed as 5'-CMP. The content of impurity F (the largest related substance except 5'-CMP) has already pass $0.1 \%$, so the chemical structure should be confirmed for studying toxic and improving the purity of the drug.

\section{EXPERIMENTAL}

Methanol, formic acid and acetonitrile for HPLC analysis was of chromatographic grade (Sigma-Aldrich, USA); ammonium acetate for HPLC analysis was of chromatographic grade (TEDIA, USA); water was purified and deionized using a Milli-Q ion-exchange filtration system (Millipore, Bedford, MA, USA); the other reagent was analytic grade. ProElut PLS SPE (500 mg) and ProElut PWC SPE (150 mg) were obtained from Dikma Technologies.

Citicoline sodium raw medicine (R1004004) was produced by Kyoma Hakko Chemical Co., LTD and obtained from SHKB Pharmaceutical Co., LTD; phosphate choline raw medicine (Y-20731009003) was obtained from Suzhou Tianma Group Tianji Bio-Pharmaceutical Co., LTD; 208 batches of citicoline sodium injection were extracted from different pharmaceutical corporations in china by SFDA; 5'-CMP (110224) was purchased from Jiakailong Science and Technology Development Co.,LTD; cytosine (069K1542), cytidine (100914316) and uridine (039K1396) reference substance were purchased from Sigma-Aldrich corporation. Citicoline sodium (140675-201005) and 5'-UMP (140735-201007) 
reference substance were purchased from the National Institute of Control of Pharmaceutical and Biological Products.

Sample preparations: Citicoline sodium injection was diluted into $2.5 \mathrm{mg} / \mathrm{mL}, 2500 \mathrm{ng} / \mathrm{mL}$ by water as sample solution. Sample solution $(2.5 \mathrm{mg} / \mathrm{mL})$ was heated at $121{ }^{\circ} \mathrm{C}$ for $2 \mathrm{~h}$ using MLS-3750 Autoclaves (SANYO, Japan) as hightemperature damaged sample solution. About $5.78 \mathrm{mg}$ cytosine reference substance was weighted using ME235S Electronic Balance (Sartorius, Germany) and diluted into $1.156 \mathrm{mg} / \mathrm{mL}$, $46.24 \mu \mathrm{g} / \mathrm{mL}, 115.6 \mathrm{ng} / \mathrm{mL}$ by water as cytosine standard solution. Cytosine standard solution $(46.24 \mu \mathrm{g} / \mathrm{mL})$ was heated at $121{ }^{\circ} \mathrm{C}$ for $2 \mathrm{~h}$ as high-temperature damaged cytosine standard solution. About $4.41 \mathrm{mg}$ cytidine reference substance was weighted and diluted into $0.882 \mathrm{mg} / \mathrm{mL}, 105.84 \mu \mathrm{g} / \mathrm{mL}, 88.2$ $\mathrm{ng} / \mathrm{mL}$ by water as cytidine standard solution. Cytidine standard solution $(105.84 \mu \mathrm{g} / \mathrm{mL})$ was heated at $121^{\circ} \mathrm{C}$ for $2 \mathrm{~h}$ as high-temperature damaged cytidine standard solution. About $2.41 \mathrm{mg}$ uridine reference substance was weighted and diluted into $241 \mu \mathrm{g} / \mathrm{mL}$ by water as uridine standard solution. Prior to injection, each sample was filtered through a $0.45 \mu \mathrm{m} \mathrm{CA}$ membrane filter.

HPLC-DAD analysis: The HPLC system consisted of a Shimadzu LC-20AD instrument with a diode array detector and managed by a LC-solution workstation (Shimadzu Corporation). The column was a ODS column $(250 \mathrm{~mm} \times 4.6$ $\mathrm{mm}$ ) with a particle size of $5 \mathrm{~mm}$ (Sigma-Aldrich, USA) maintained at $30{ }^{\circ} \mathrm{C}$. The mobile phase was A-phosphate buffer [mix the potassium dihydrogen phosphate solution $(0.1$ $\mathrm{mol} / \mathrm{L})$ and the tetrabutylammonium hydroxide solution $(10$ $\mathrm{mmol} / \mathrm{L}$ ) equally, adjust the $\mathrm{pH}$ value of the mixture at 4.7 by phosphate] and B-methanol $(96: 4, \mathrm{v} / \mathrm{v})$ at a flow rate of 0.8 $\mathrm{mL} / \mathrm{min}$. The UV spectra were recorded between $210 \mathrm{~nm}$ and $400 \mathrm{~nm}$.

HPLC-MS/MS analysis: The HPLC system consisted of a Agilent 1200 instrument and it was interfaced with a Agilent 6410 QQQ mass spectrometer (Agilent Technologies, Pal Alto, CA, USA). The column was a ODS column (100 mm $\times 2.1 \mathrm{~mm}$ ) with a particle size of $3.5 \mathrm{~mm}$ (Agilent, USA) maintained at $30{ }^{\circ} \mathrm{C}$. The mobile phase was A-ammonium acetate solution $(5 \mathrm{mM})$ and B-acetonitrile $(95: 5, \mathrm{v} / \mathrm{v})$ at a flow rate of 0.3 $\mathrm{mL} / \mathrm{min}$. Mass spectrometry operating conditions: Electrospray ionization source (ESI) was applied and operated in positive ionization mode, drying gas temperature was at $350^{\circ} \mathrm{C}$, nitrogen flow was $9.0 \mathrm{~L} / \mathrm{min}$, nebulizer pressure $40 \mathrm{psi}$, capillary voltage was set at $4.0 \mathrm{KV}$, scan spectra from $\mathrm{m} / \mathrm{z} 100$ to 1000 . Full scan, selected ion monitoring (SIM) and product ion scan were used.

Synthesis of uridine diphosphate choline $\mathrm{e}^{17,18}$ : Weigh phosphorylcholine $12.5 \mathrm{~g}$ in a conical flask, adding $40 \mathrm{~mL}$ DMF. Put the conical flask in $70{ }^{\circ} \mathrm{C}$ water bath until syruplike. Weigh $10 \mathrm{~g}$ of paratolue ulfonyl chloride in a conical flask, adding $15 \mathrm{~mL}$ DMF and the above syrup-like, shaking the conical flask at room temperature until phosphorylcholine dissolve absolutly. Add 1 g 5'-UMP, stirred 3-4 h at room temperature, then the reaction end. Dissolve it in $500 \mathrm{~mL}$ distilled water and the synthetic sample was obtained. Precise amount of $1 \mathrm{~mL}$, diluted 1000 times with water as uridine diphosphate choline reference standard.

\section{RESULTS AND DISCUSSION}

The related substances of citicoline sodium injection were analyzed by HPLC-DAD (Fig. 1).

Chemical structure of impurity A,B analysis: Citicoline is a diphosphate, so it would be easily degraded into phosphocholine, choline, 5'-CMP, cytosine, cytidine and other impurities. The total ion chromatogram (TIC) of sample solution obtained from the LC-MS/MS system in positive mode and the extracted ion chromatograms (EIC) of three peaks is shown in Fig. 2. The three peaks were named as peak 1, 2, 3 according to retention time. $\mathrm{M}^{+} 112.1,184.1,264,378,489.1,511.2$, 527.1 were shown in EIC of peak 1 and $\mathrm{M}^{+}$489.1, 511.2, 527.1 were $[\mathrm{M}+\mathrm{H}]^{+},[\mathrm{M}+\mathrm{Na}]^{+},[\mathrm{M}+\mathrm{K}]^{+}$of citicoline. $\mathrm{M}^{+} 104.1$ was shown in EIC of peak 2. $\mathrm{M}^{+} 104.1,112.1,244.1,266.1,282.1$ were shown in EIC of peak 3. The tandem mass (MS/MS) spectrum of selected ion $(\mathrm{m} / \mathrm{z}, 489.1$, citicoline) is shown in Fig. 3.

Compared with molecular weight and MS/MS spectra of cytosine and cytidine standard solution, cytosine and cytidine were confirmed as two impurities in sample solution. The retention time and UV spectra of impurity A,B were corresponded with cytosine and cytidine standard solution, indicating that impurity A,B were cytosine and cytidine, respectively. Related patterns are shown in Figs. 4-7. The structure, quasimolecular ions and their product ions of three impurities are shown in Table-1.

The content of impurity C,D,G were too little to be analyzed, so the molecular weight and MS/MS spectra were unknown and the structures were speculated preliminary according to the UV spectra. The UV spectra of impurity A,B,C,D,E were similar. Impurity E has known as 5'-UMP and impurity A,B were identified as cytosine and cytidine in this study, so impurity C,D were speculated having the same cytosine nucleus. The UV spectrum of impurity G was significantly different.

Analysis of impurity F: Because of weak acidity and strong polarity, ion-pair reagent and phosphate buffer were used to increase retention time and improve peak shape of citicoline sodium in HPLC-DAD analysis. The impurity F was high-temperature degraded product of citicoline, so it was collected corresponding retention time when high-temperature damaged sample solution was analyzed by HPLC-DAD for several times. The collected impurity F solution has a lot of tetrabutyl-ammonium hydroxide and phosphate buffer, it would not be used in LC-MS system. So, demineralized impurity F solution was obtained from the collected impurity F solution by DIKMA ProElut PLS and PWC SPE ${ }^{19}$. The EIC and selected ion $(\mathrm{m} / \mathrm{z}$ 490.0) MS/MS spectra of demineralized impurity $\mathrm{F}$ solution is shown in Fig. 8. The molecular weight of impurity F was 489.1.

Presumed structure of impurity F: Compared with citicoline, the UV spectrum of impurity F was significantly different. A allosteric of cytosine or a conjugated double bonds was produced after a dehydrated condensation of ribose may cause the phenomenon. In Figs. 9 and 10, the chromatogram and UV spectra of high-temperature damaged cytosine/cytidine standard solution by HPLC-DAD were shown. After high- 

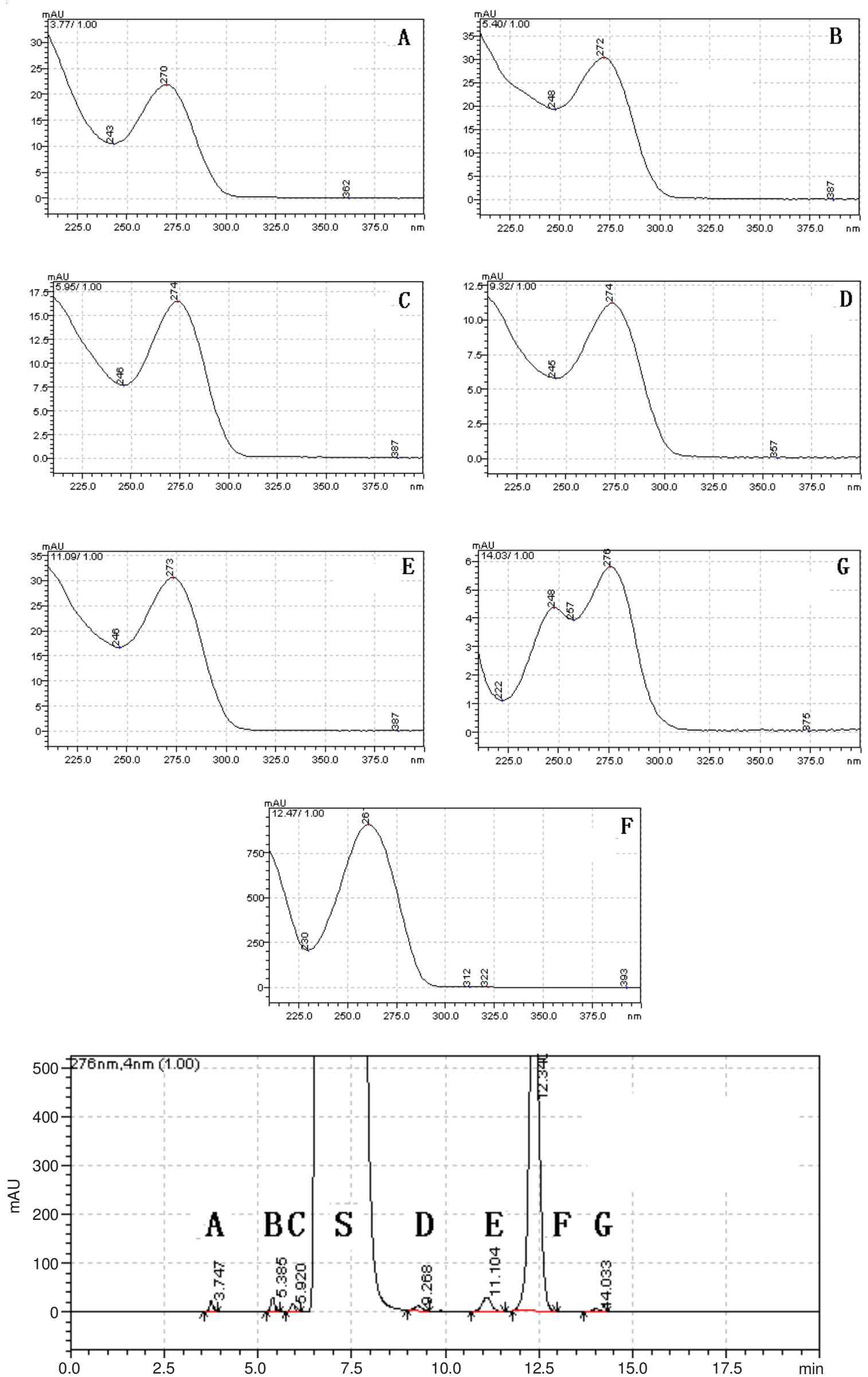

Fig. 1. Chromatogram of sample solution at $276 \mathrm{~nm}$ and UV spectra of seven chromatographic peak 

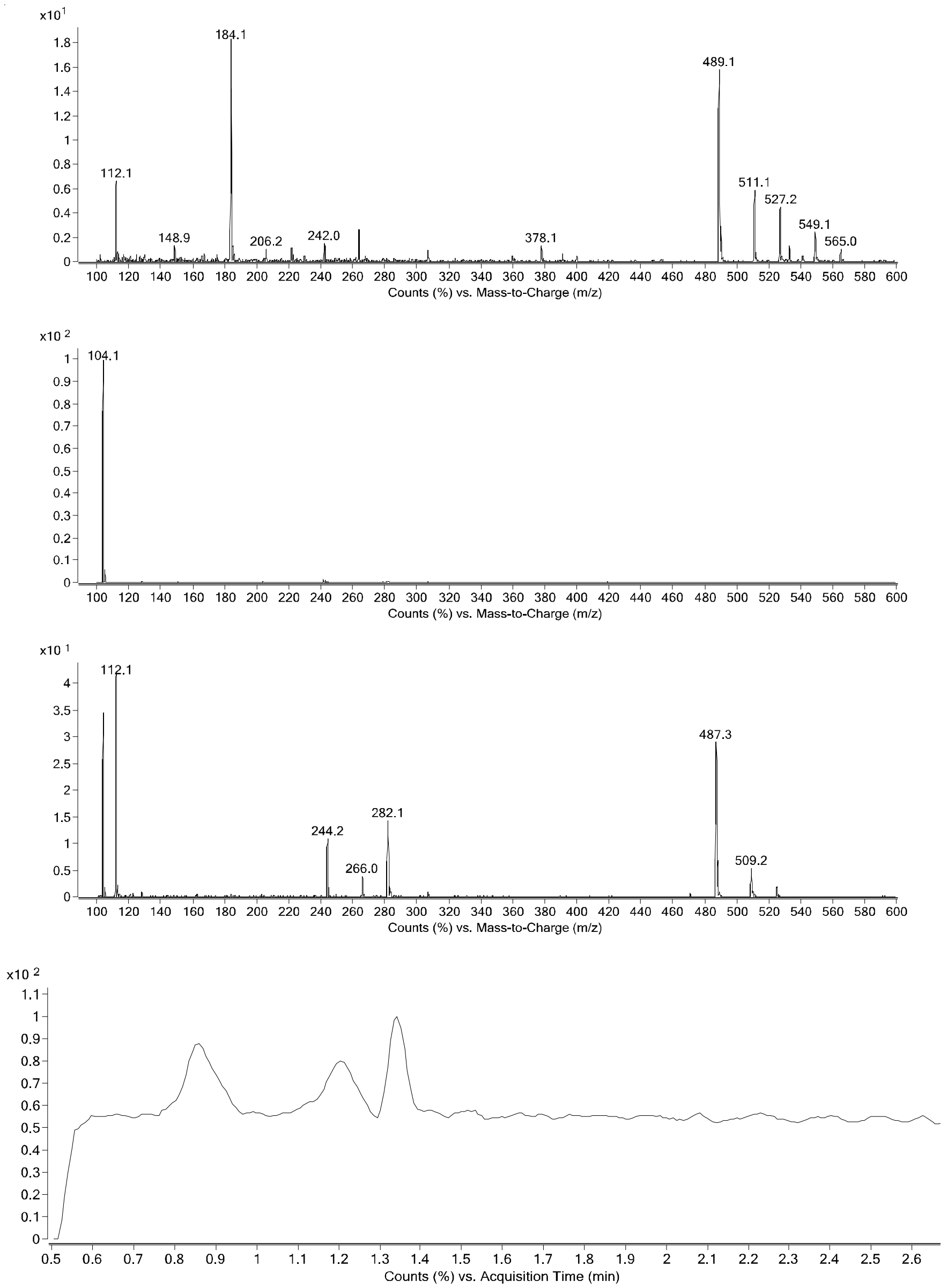

Fig. 2. Total ion chromatography of sample solution and the extracted ion chromatogram of three peaks 


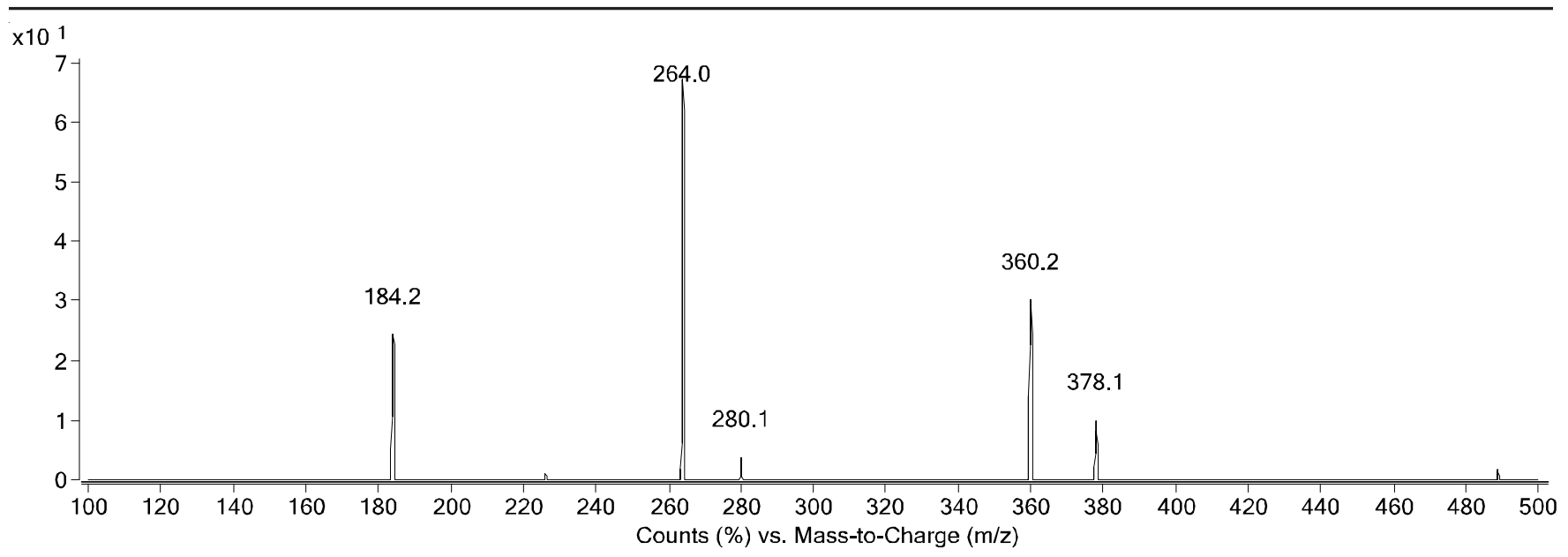

Fig. 3. The MS/MS spectrum of selected ion ( $m / z$ 489.1, citicoline)

$\mathrm{x} 10^{2}$

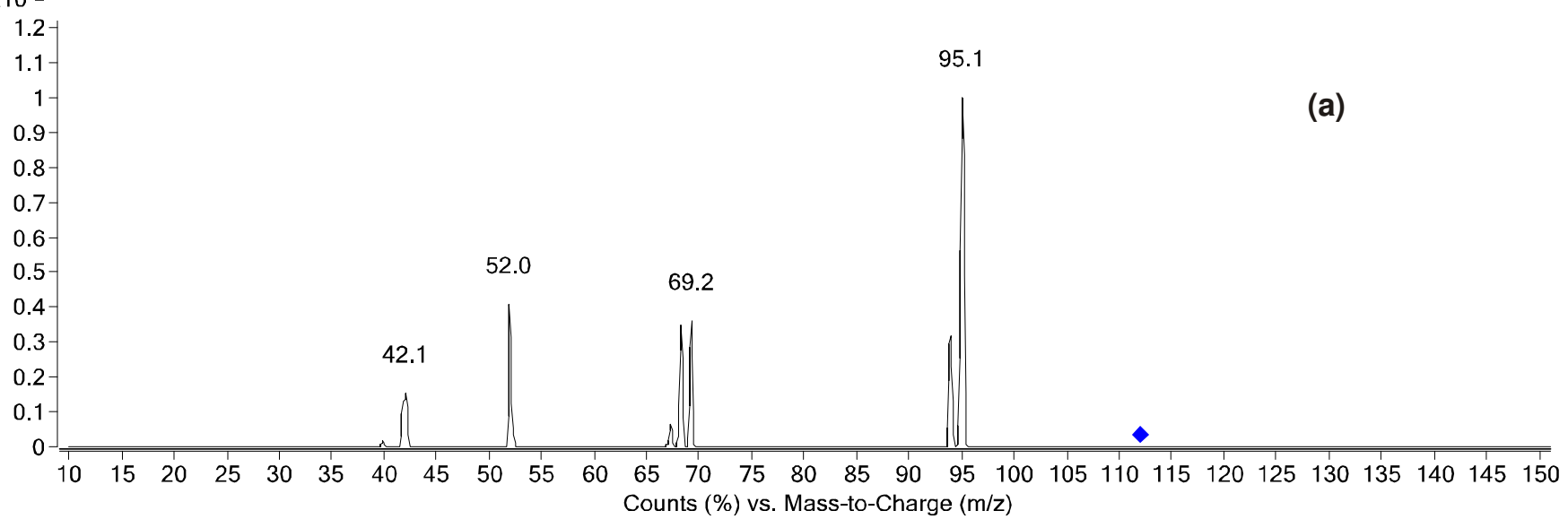
$\mathrm{x} 10^{2}$

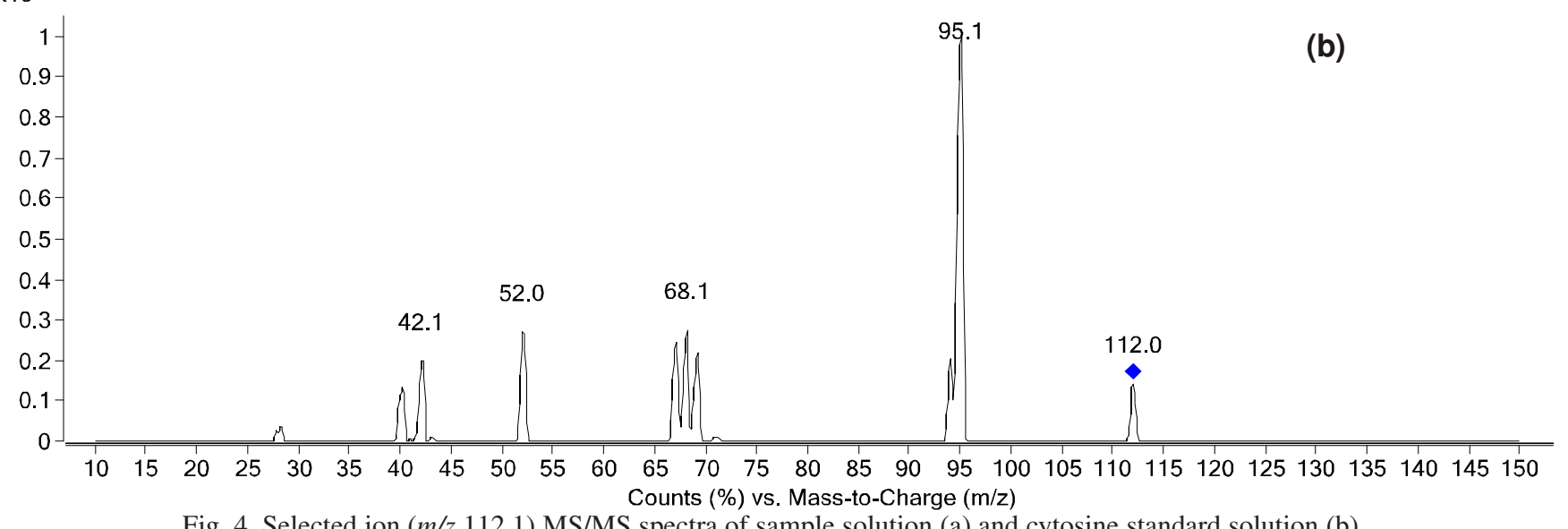

Fig. 4. Selected ion $(\mathrm{m} / \mathrm{z}, 112.1) \mathrm{MS} / \mathrm{MS}$ spectra of sample solution (a) and cytosine standard solution (b)
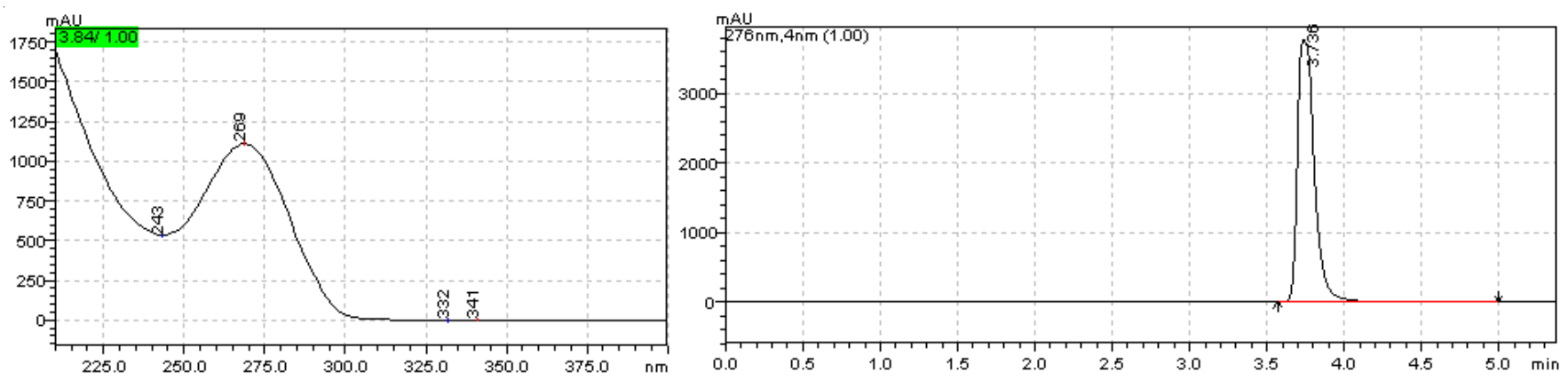

Fig. 5. Chromatogram and UV spectrum of cytosine standard solution 

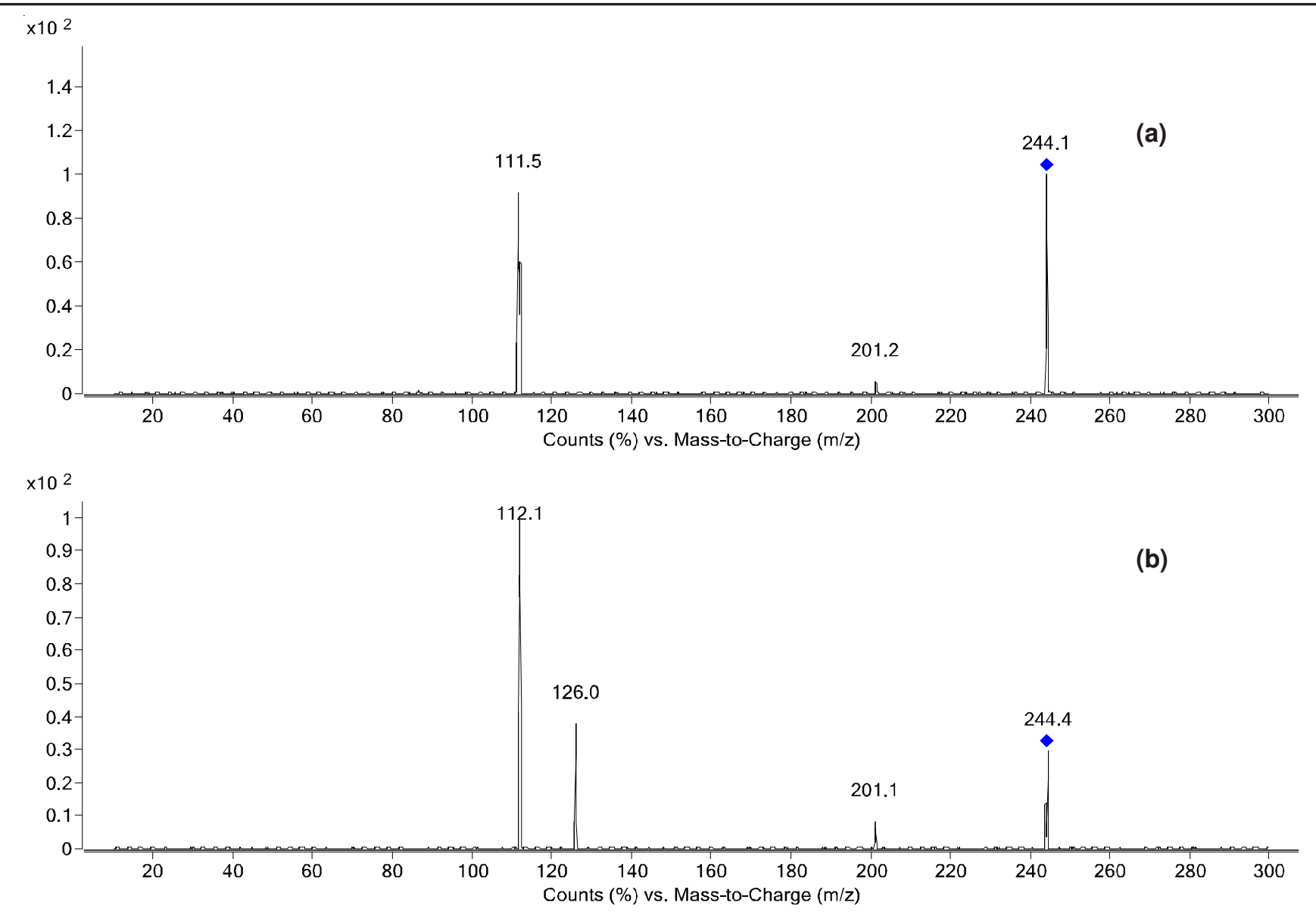

Fig. 6. Selected ion ( $m / z$ 244.1) MS/MS spectra of sample solution (a) and cytidine standard solution (b)

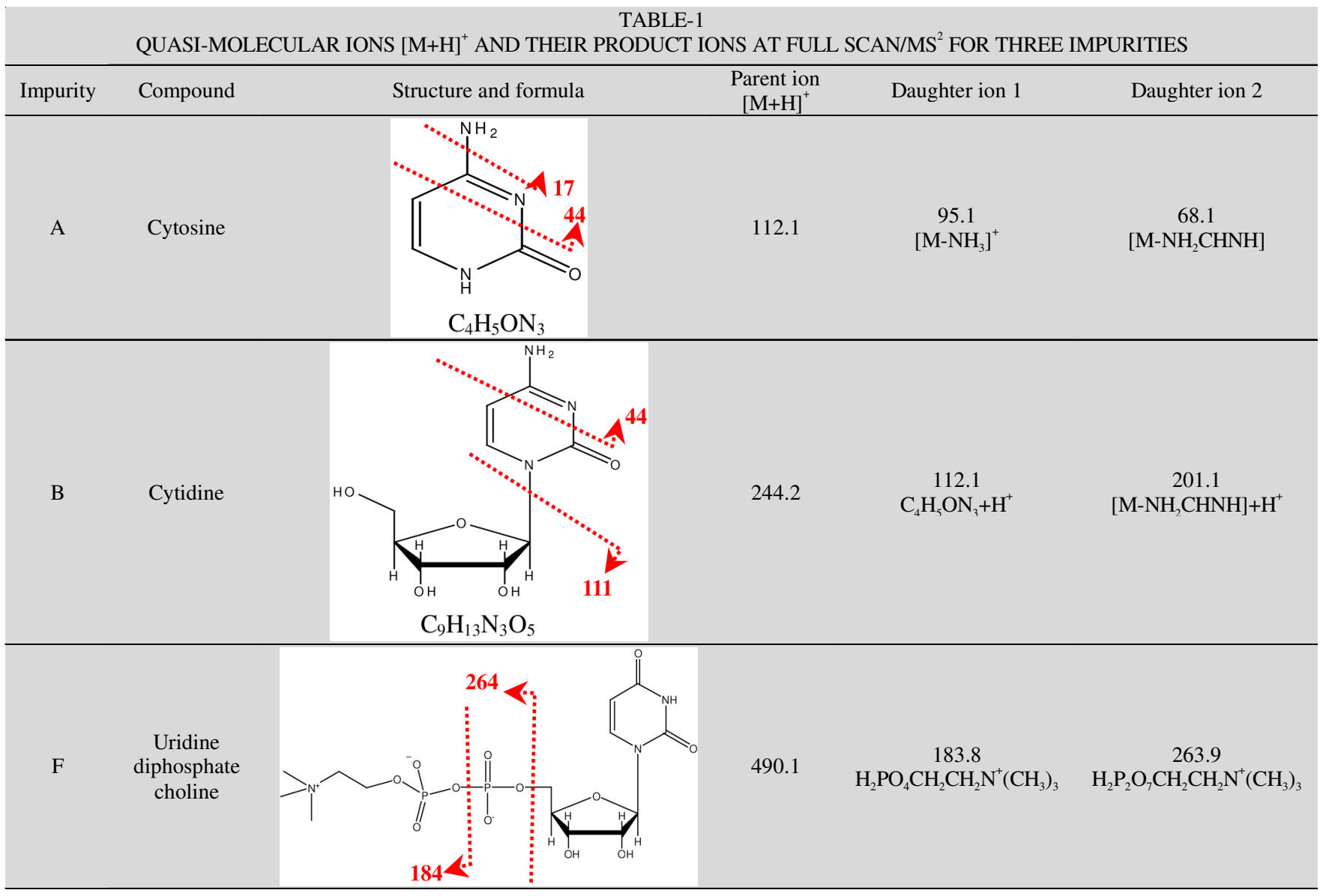



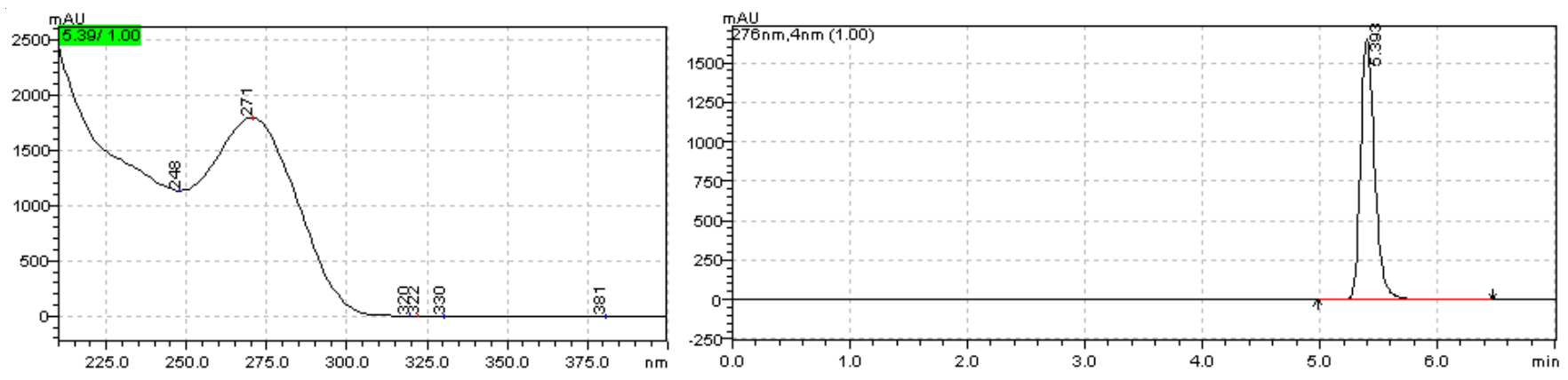

Fig. 7. Chromatogram and UV spectrum of cytidine standard solution
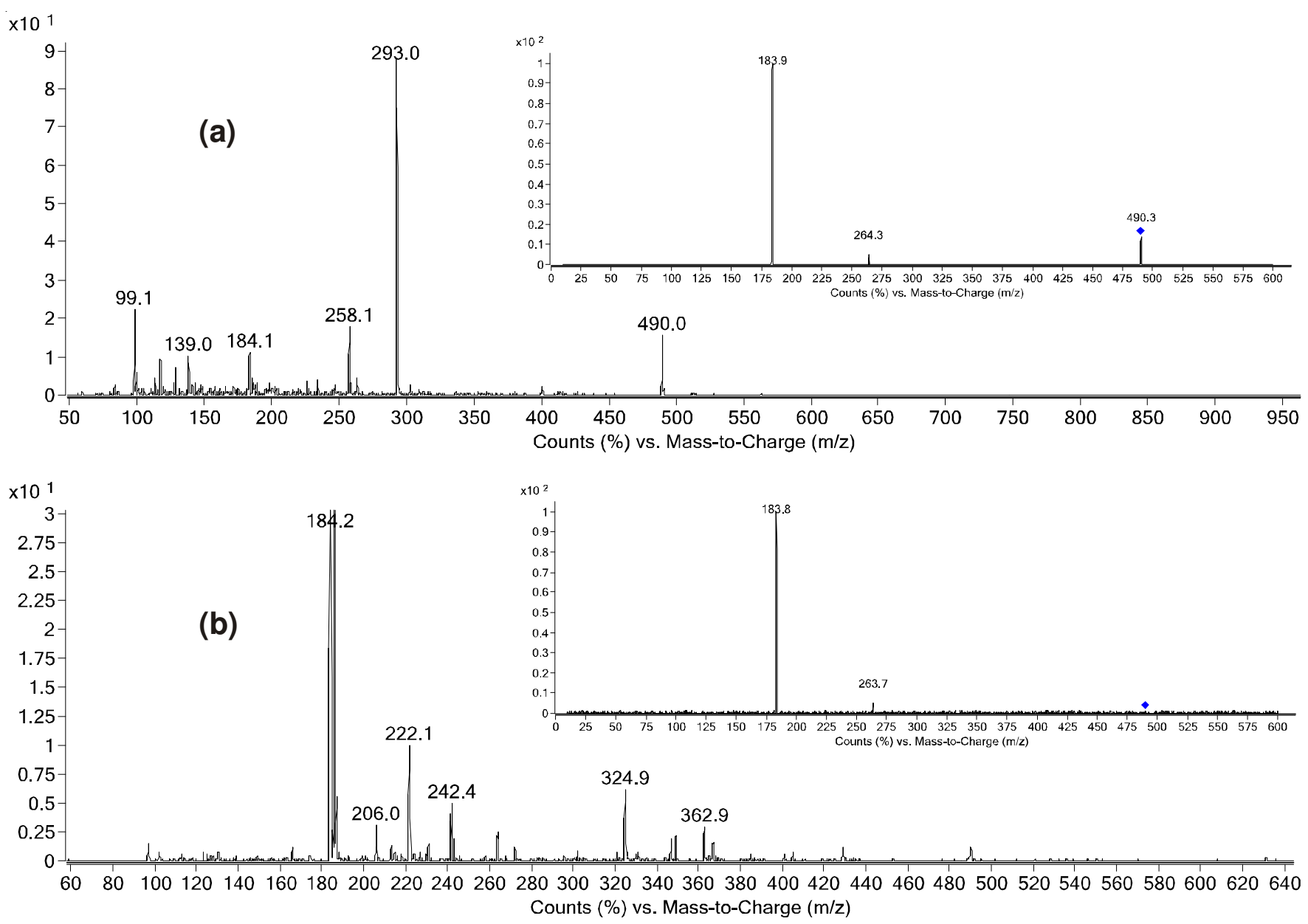

Fig. 8. Extracted ion chromatogram and selected ion ( $\mathrm{m} / \mathrm{z}$ 490.1) MS/MS spectra of impurity F (a) and uridine diphosphate choline standard solution (b)
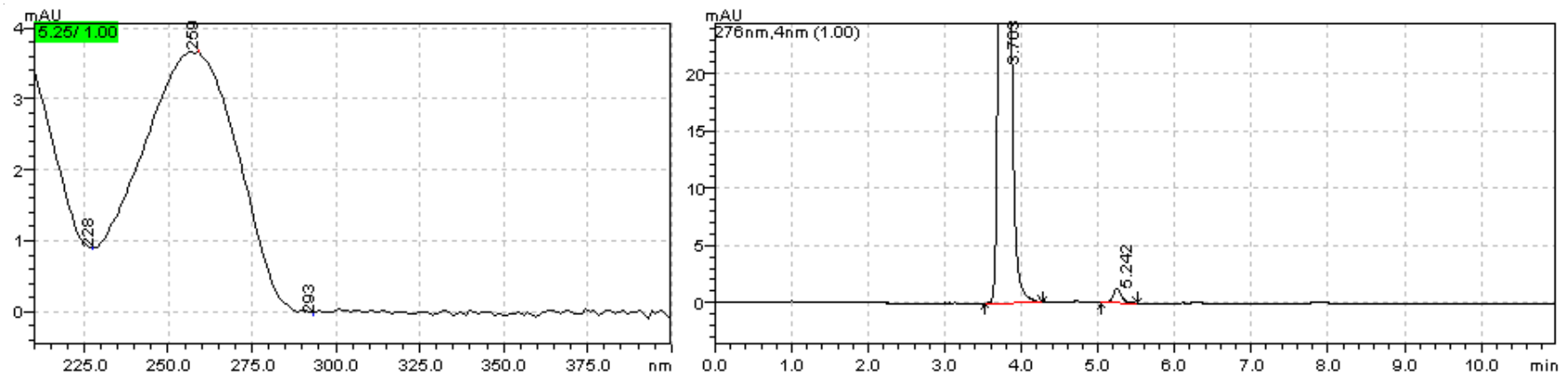

Fig. 9. Chromatogram and UV spectrum of high-temperature damaged cytosine standard solution 

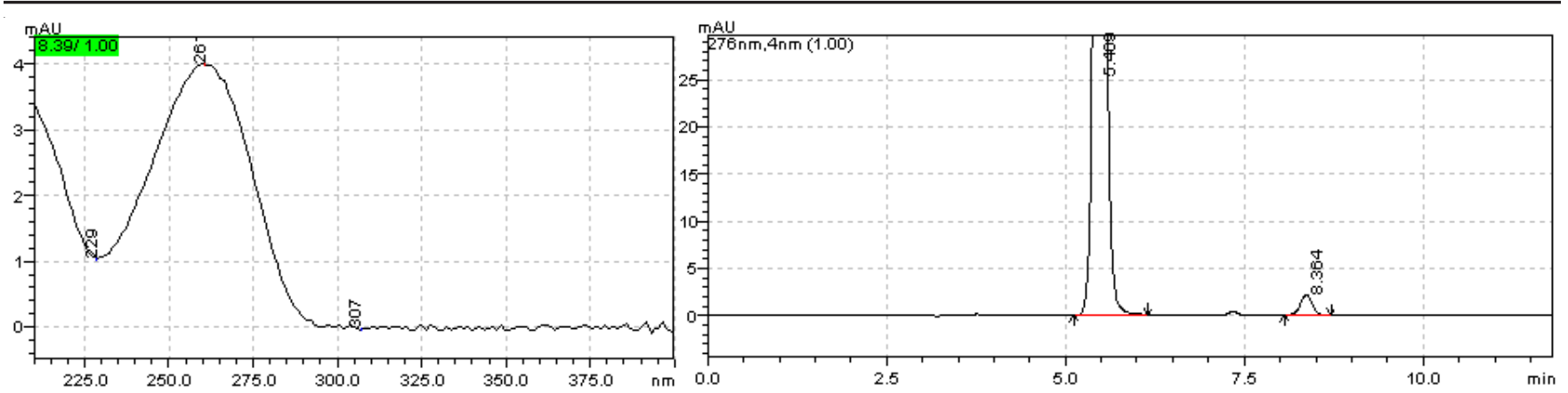

Fig. 10. Chromatogram and UV spectrum of high-temperature damaged cytidine standard solution
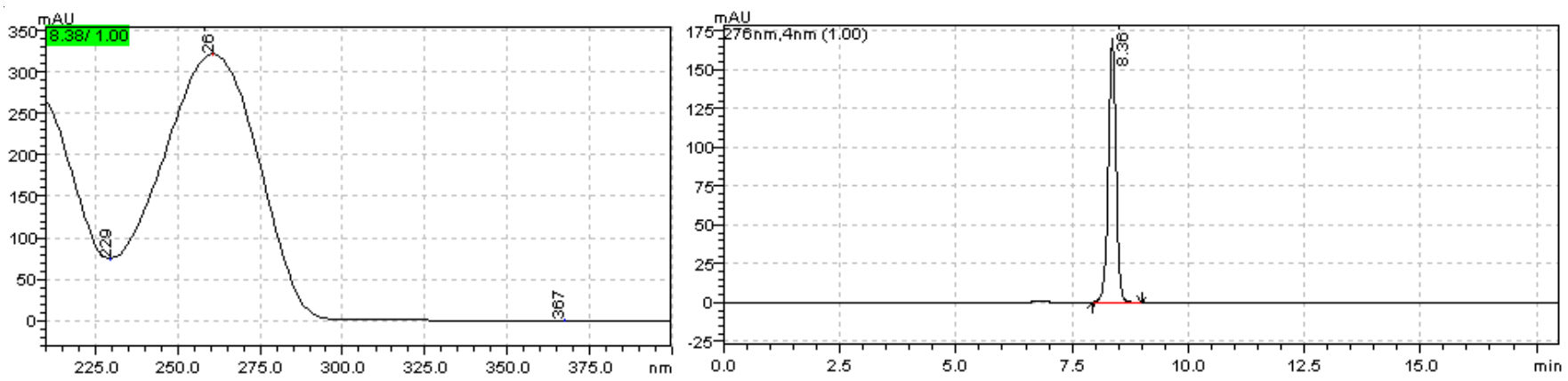

Fig. 11. Chromatogram and UV spectrum of uridine standard solution
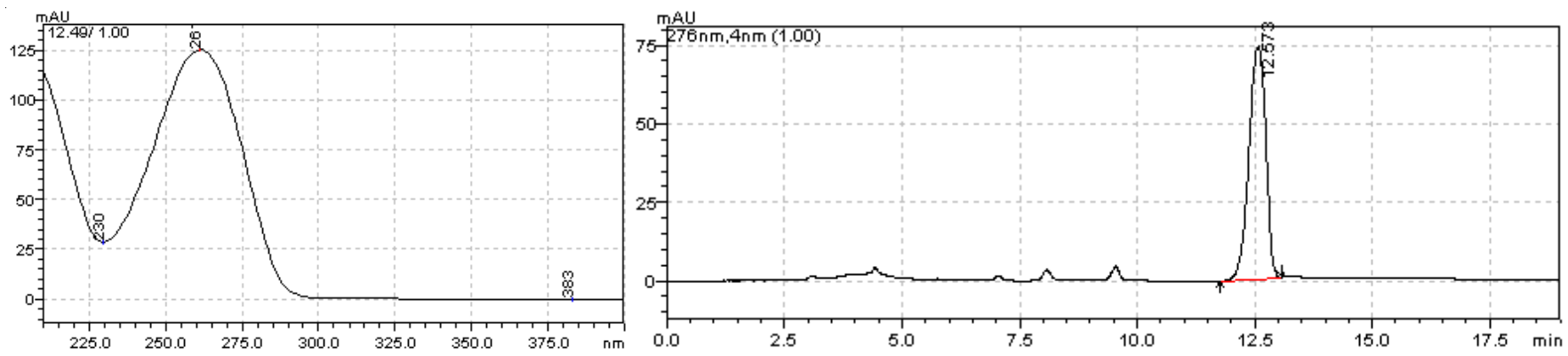

Fig. 12. Chromatogram and UV spectrum of uridine diphosphate choline standard solution

temperature damaged, A few degradation products of cytosine and cytidine were produced. The UV spectra of degradation products were similar, indicating cytosine was changed, while the ribose didn't changed. In Fig. 11, the chromatogram and UV spectrum of uridine standard solution by HPLC-DAD was shown. Compared with uridine, the retention time and UV spectrum of degraded product of cytidine were the same, so that a few cytidine could be transfered to uridine after hightemperatured damaged. The UV spectra of impurity $\mathrm{F}$ and uridine reference standard were the same. Therefore, we could speculate that uridine was one part of impurity $\mathrm{F}$ and impurity $\mathrm{F}$ was presumed as uridine diphosphate choline.

The chromatogram and UV spectrum of uridine diphosphate choline standard solution were shown in Fig. 12. The EIC and selected ion $(\mathrm{m} / \mathrm{z}$ 490.1) MS/MS spectra of uridine diphosphate choline standard solution and impurity $\mathrm{F}$ were shown in Fig. 8. Compared with impurity F, the retention time, UV spectra and the selected ion $(\mathrm{m} / \mathrm{z}$ 490.1) MS/MS spectra of uridine diphosphate choline standard solution were the same.

\section{REFERENCES}

1. K.J. D'Orlando and B.W. Sandage Jr, Neurol. Res., 17, 281 (1995).

2. I. López-Coviella, J. Agut, V. Savci, J.A. Ortiz and R.J. Wurtman, J. Neurochem., 65, 889 (1995).
3. J.J. Secades and G. Frontera, Methods Find Exp. Clin. Pharmacol., 17 Suppl B, 1 (1995).

4. G.B. Weiss, Life Sci., 56, 637 (1995).

5. X.A. Alvarez, M. Laredo, D. Corzo, L. Fernández-Novoa, R. Mouzo, J.E. Perea, D. Daniele and R. Cacabelos, Methods Find Exp. Clin. Pharmacol., 19, 201 (1997).

6. P.A. Spiers, D. Myers, G.S. Hochanadel, H.R. Lieberman and R.J. Wurtman, Arch. Neurol., 53, 441 (1996).

7. J. Bruhwyler, J. Vandorpe and J. Geczy, Curr. Ther. Res. Clin. Exp., 58, 309 (1997).

8. W.M. Clark, S.J. Warach, L.C. Pettigrew, R.E. Gammans and L.A. Sabounjian, Neurology, 49, 671 (1997).

9. W.M. Clark, B.J. Williams, K.A. Selzer, R.M. Zweifler, L.A. Sabounjian and R.E. Gammans, Stroke, 30, 2592 (1999).

10. C.E. Dixon, X.C. Ma and D.W. Marion, J. Neurotrauma, 14, 161 (1997).

11. T. Hazama, T. Hasegawa, S. Ueda and A. Sakuma, Int. J. Neurosci., 11, 211 (1980).

12. K. Overgaard, T. Sereghy, G. Boysen, H. Pedersen and N.H. Diemer, Stroke, 23, 1167 (1992).

13. Y. Tazaki, F. Sakai, E. Otomo, T. Kutsuzawa, M. Kameyama, T. Omae, M. Fujishima and A. Sakuma, Stroke, 19, 211 (1988).

14. M. Fresta and G. Puglisi, Life Sci., 61, 1227 (1997).

15. R. Ozay, A. Bekar, H. Kocaeli, N. Karli, G. Filiz and I.H. Ulus, Surg. Neurol., 68, 615 (2007).

16. Pharmacopoeia of the People's Republic of China, Vol. II, pp. 602-603 (2010).

17. K. Kikugawa, M. Ichino and T. Kawashima, Chem. Pharm. Bull., 19, 2466 (1971).

18. F.-C. Gu and L.-Y. Yang, Fajiao Keji Tongxun, 36, 9 (2007).

19. SPE Principles and Techniques, Dikma Technologies, edn. 2. 